\title{
Solvothermal synthesis of octahedral nickel oxides nanoparticles
}

\author{
Lijuan $\operatorname{Wan}^{1,2^{*}}$ \\ ${ }^{1}$ Nanjing Communications Institute of Technology, Nanjing 211188, China; \\ 2 Jiangsu Engineering Technology Research Center for Energy Conservation and Emission \\ Reduction of Transportation, Nanjing 211188, China. \\ *bartty_ym@163.com.
}

Keywords: nickel oxide; solvothermal; morphology

\begin{abstract}
Controlling the morphology of inorganic nanomaterials attracts more and more attention, due to the influence of morphology on the material properties and application. The nanostructures of nickel oxide $(\mathrm{NiO})$ has attracted great interest because of its unique physical and chemical properties for a broad range of applications. A novel and facile synthesis route for the manufacture of octahedral nickel oxide nanoparticles is reported, utilizing nickel chloride as the inorganic source by the solvothermal method in an environmentally benign alcohol-water mixture. X-ray powder diffraction (XRD), the Brunauer-Emmett-Teller method and scanning electron microscopy (SEM) were used to characterize the as-prepared nickel oxide products. The possible formation mechanism of the octahedral nickel oxide nanoparticles was discussed.
\end{abstract}

\section{Introduction}

There has been increasing interest in the controlled synthesis of inorganic micro- and nanostructures, and in current material synthesis, the ability to tune the structure, size and morphology of inorganic materials is an important goal, and. The nanostructured nickel hydroxide and nickel oxide arouse increasing attention for unique physical and chemical properties. As a p-type semiconductor with unique optical and magnetic properties, nickel(II) oxide (NiO) is a promising material with potential applications in electrochromic windows [1]. $\mathrm{NiO}$ is also a valuable semiconductor, catalytic and antiferromagnetic material used in different fields, for instance, photocatalysis [2], dye-adsorbed NiO solar cells (pDSCs) [3], pseudocapacitor [4], sensors such as non-enzymatic glucose sensor [5].

$\mathrm{NiO}$ has attracted great interest because of its favorable physicochemical properties. Especially, due to the large chemical and thermal stability, nanostructured $\mathrm{NiO}$, shows great promise for the use in energy conversion applications. There are several reports on the pseudocapcitive performance of different $\mathrm{NiO}$ based nanostructures, such as nanowires [6], nanocolumns [7], nanoballs [8], nanotubes [9] and etc. Furthermore, a facile method is used to synthesize NiO microspheres with different nanoscale building blocks via a hydrothermal route [10]. The porous $\mathrm{NiO}$ nanocolumns and nanosheets are obtained by the calcination of the $\mathrm{Ni}(\mathrm{OH}) 2$ nanocolumns [11]. NiO nanodiscs and nanoflowers have been synthesized through non-basic solution eco-routes [12].

For the synthesis of nickel oxide nanoparticles, some mild and environmentally benign methods become more and more important. It has been reported that single-crystalline hollow NiO crystals with well defined octahedral morphology were synthesized by using a template-assisted carbothermal method [13]. Recently, by solution combustion method, octahedral nickel oxide nanoparticles were synthesized [14]. By a simple hydrothermal technique, NiO powders with a spherical morphology were synthesized using organic surfactants as templates and urea as the hydrolysis controlling agent [15]. In this study, the octahedral nickel oxide nanoparticles have been prepared through the facile solvothermal route in an environmentally benign alcohol-water mixture. The growth mechanism of the octahedral nickel oxide nanoparticles was also investigated. 


\section{Experimental Section}

\subsection{Materials}

Triblock copolymer Pluronic F-127 (EO106PO70EO106, MW=12600, product no. P2443-250G) was purchased from Aldrich and used as received without further purification.

\subsection{Synthesis of $\mathrm{NiO}$}

In a typical experiment, isotropic solutions were prepared by dissolving $\mathrm{Ni}(\mathrm{NO} 3) 2 \cdot 6 \mathrm{H} 2 \mathrm{O}$ or $\mathrm{NiCl} \cdot 6 \mathrm{H} 2 \mathrm{O}$ (0.1 mol) and block copolymer F-127 (EO106PO70EO106) (0.9 g) in EtOH (23 mL), $\mathrm{H} 2 \mathrm{O}(20 \mathrm{~mL})$ and $26-28 \% \mathrm{NH} 3 \cdot \mathrm{H} 2 \mathrm{O}(4.5 \mathrm{~mL})$. The solution was stirred at $70{ }^{\circ} \mathrm{C}$ for $1 \mathrm{~h}$, then it was transferred into a Teflon-lined stainless steel autoclave and heated at $150{ }^{\circ} \mathrm{C}$ for $16 \mathrm{~h}$. To remove the surfactant, the as-prepared products were washed with water, and then the sample was dried and calcined at $450{ }^{\circ} \mathrm{C}$ for $4 \mathrm{~h}\left(1{ }^{\circ} \mathrm{C}\right.$ min-1 under air).

\subsection{Characterization}

The crystalline phase and orientation of products were identified by X-ray diffraction (XRD) on a Rigaku D/MAX-Ultima III X-ray diffractometer with $\mathrm{Cu} \mathrm{K} \alpha$ radiation $(\lambda=0.154 \mathrm{~nm}, 40 \mathrm{kV}, 40 \mathrm{~mA})$ and a scan rate of $10^{\circ} \cdot \mathrm{min}-1$. The microstructure of the as-prepared products was characterized by a field emission scanning electron microscopy (FE-SEM, S4800, Hitachi Ltd.) with an electron accelerating voltage of $15 \mathrm{kV}$. The specific surface area of the as-prepared $\mathrm{NiO}$ powders was obtained on a Micromeritics TriStar 3000 instrument and the Brunauer-Emmett-Teller (BET) equation was used to calculate the specific surface area.

\section{Results and Discussion}

Fig. 1 shows the structural characteristics of the sample prepared through the solvothermal route by using nickel chloride in the starting solution investigated by XRD. The diffraction peaks typical to $\mathrm{NiO}$ are clearly observed, which agree with those of standard NiO of cubic structure (JCPDS Card No. 47-1049). There are no other peaks observed in the XRD result except for the diffraction peaks of $\mathrm{NiO}$, which means single-phase $\mathrm{NiO}$ can be obtained route in this system through the solvothermal route.

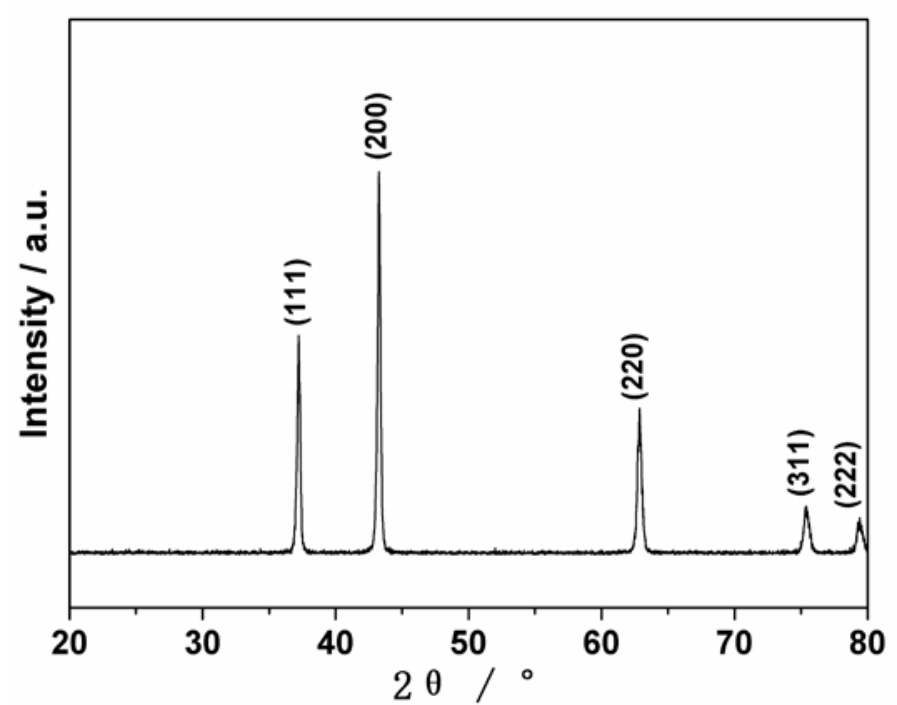

Fig. $1 \mathrm{XRD}$ pattern of the product calcined at $450{ }^{\circ} \mathrm{C}$ synthesized by using $\mathrm{NiCl} 2 \cdot 6 \mathrm{H} 2 \mathrm{O}$ as precursor.

The morphology of the as-prepared $\mathrm{NiO}$ sample was characterized by FE-SEM and the images are shown in Fig. 2. From Fig. 2, the low-magnification SEM image (see Fig. 2a) of the as-prepared $\mathrm{NiO}$ through solvothermal method by using $\mathrm{NiCl} 2 \cdot 6 \mathrm{H} 2 \mathrm{O}$ as precursor shows a uniform octahedral morphology in a large range. The octahedral structure of the as-prepared $\mathrm{NiO}$ sample can be clearly observed by the high-magnification SEM image (see Fig. 2b). The BET surface area of the as-prepared $\mathrm{NiO}$ sample $5.02 \mathrm{~m} 2 \mathrm{~g}-1$. 


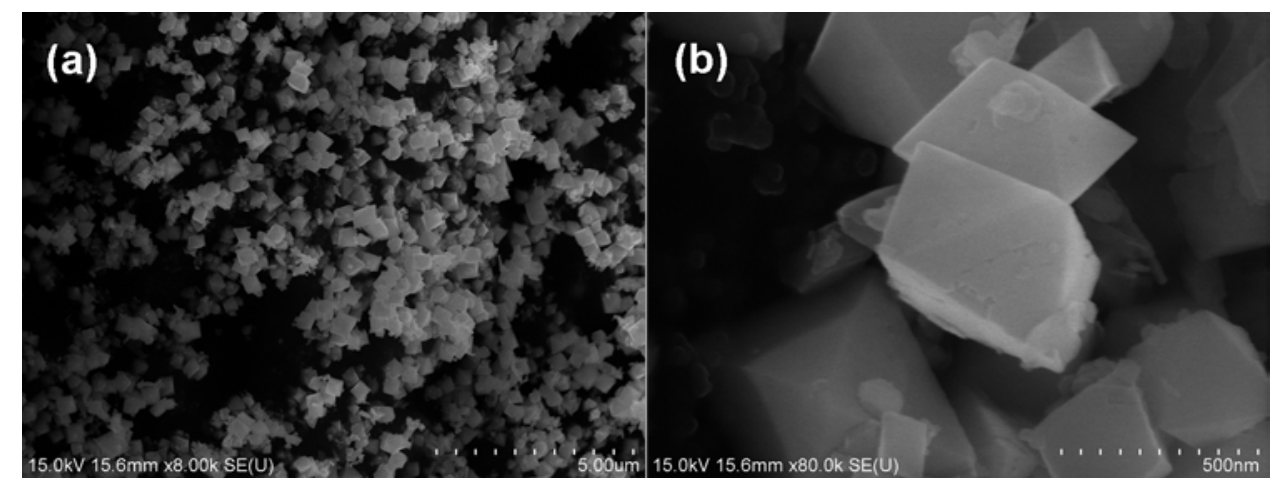

Fig. 2 SEM images of the product calcined at $450{ }^{\circ} \mathrm{C}$ synthesized by using $\mathrm{NiCl} 2 \cdot 6 \mathrm{H} 2 \mathrm{O}$ as precursor.

Fig. 3 shows the low- and high magnification SEM images of the as-prepared NiO by using $\mathrm{Ni}(\mathrm{NO} 3) 2 \cdot 6 \mathrm{H} 2 \mathrm{O}$ in the starting solution. From Fig. 3a, the low-magnification SEM image shows the $\mathrm{NiO}$ particles morphology. From Fig. 3b, the high-magnification SEM image shows the morphology of the $\mathrm{NiO}$ particles is plate-like shape.

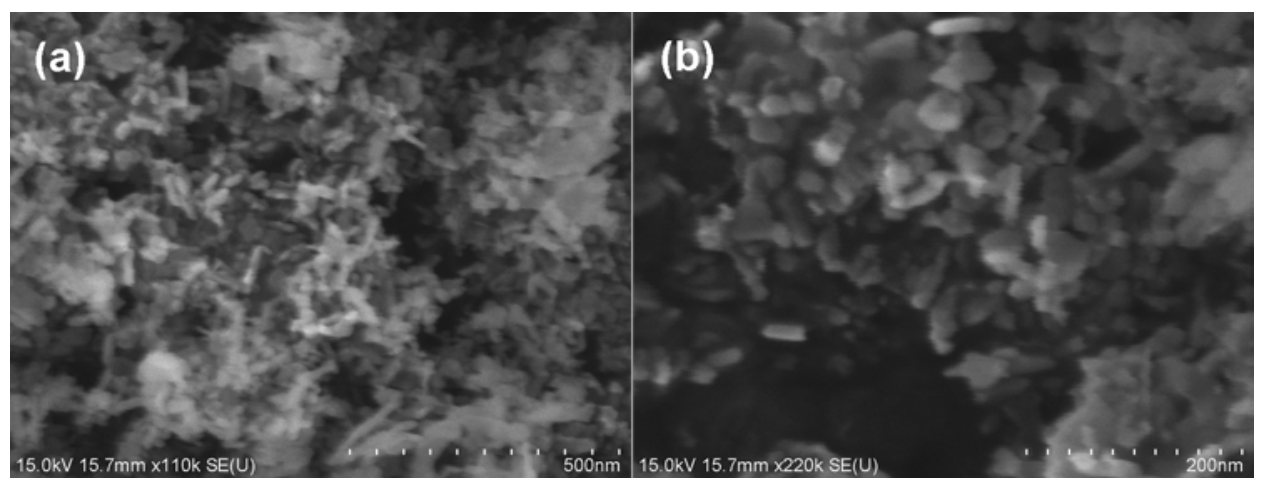

Fig. $3 \mathrm{SEM}$ images of the product calcined at $450{ }^{\circ} \mathrm{C}$ synthesized by using $\mathrm{Ni}\left(\mathrm{NO}_{3}\right)_{2} \cdot 6 \mathrm{H}_{2} \mathrm{O}$ as precursor.

In principle, in a crystal growth process in solution, the nucleation and growth are affected by the intrinsic crystal structure and the external conditions including the kinetic energy barrier, time, temperature, capping molecules and etc. Due to the same synthesis condition, the existence of $\mathrm{Cl}-$ in the starting solution may be the main effect for the preferential adsorption on some specific face to stabilize non-equilibrium morphologies by changing the relative growth rates of different crystal. It has been reported that smallest-sized $\mathrm{Cl}-$ ions with a prominent complexing ability can be easily grafted on the $\alpha-\mathrm{Ni}(\mathrm{OH}) 2$ nuclei and lower their surface energy [16]. Thus, the formation of octahedral $\mathrm{NiO}$ in this system may be attributed to the adsorption of $\mathrm{Cl}-$ on some specific face.

\section{Conclusions}

In summary, octahedral $\mathrm{NiO}$ is synthesized through solvothermal route using nickel chloride as the inorganic source in an environmentally benign alcohol-water mixture. The growth mechanism of octahedral $\mathrm{NiO}$ has been investigated. It is supposed that the formation of octahedral $\mathrm{NiO}$ may be attributed to the adsorption of $\mathrm{Cl}-$ on some specific face. $\mathrm{NiO}$ with the octahedral structure may be used in many ways such as the solar cells.

\section{Acknowledgement}

This work was supported by a grant from Scientific Research Project of Nanjing Communications Institute of Technology (JY1304), High-level Scientific Research Foundation for the introduction of talent of Nanjing Communications Institute of Technology and Qing Lan Project of Nanjing 
Communications Institute of Technology.

\section{References}

[1] G.A. Niklasson, C.G. Granqvist, , Electrochromics for smart windows: thin films of tungsten oxide and nickel oxide, and devices based on these, J. Mater. Chem. 2007, 17, 127-156.

[2] Z.Y. Zhang, C.L. Shao, X.H. Li, C.H. Wang, M.Y. Zhang, Y.C. Liu, Electrospun Nanofibers of p-Type NiO/n-Type ZnO Heterojunctions with Enhanced Photocatalytic Activity, ACS Appl. Mater. Interfaces, 2010, 2, 2915-2923.

[3] S. Mori, S. Fukuda, S. Sumikura, Y. Takeda, Y. Tamaki, E. Suzuki, T. Abe, Charge-Transfer Processes in Dye-Sensitized NiO Solar Cells, J. Phys. Chem. C 2008, 112, 16134-16139.

[4] A.K. Singh, D. Sarkar, G.G. Khan, K. Mandal, Hydrogenated NiO Nanoblock Architecture for High Performance Pseudocapacitor, ACS Appl. Mater. Interfaces 2014, 6, 4684-4692.

[5] W. Wang, Z.Y. Li, W. Zheng, B. Dong, S.Y. Li, C. Wang, A Novel Non-Enzymatic Glucose Sensor Based on Nickel (II) Oxide Electrospun Nanofibers, Journal of Nanoscience and Nanotechnology, 2010, 10, 7537-7540.

[6] A.K. Singh, D. Sarkar, G.G Khan, K. Mandal, Unique hydrogenated Ni/NiO core/shell 1D nano-heterostructures with superior electrochemical performance as supercapacitors. J. Mater. Chem. A 2013, 1, 12759-12767.

[7] X. Zhang, W. Shi, J. Zhu, W. Zhao, J. Ma, S. Mhaisalkar, T.L. Maria, Y. Yang, H. Zhang, H.H. Hng, Q. Yan, Synthesis of porous NiO nanocrystals with controllable surface area and their application as supercapacitor electrodes. Nano Res. 2010, 3, 643-652.

[8] J.W. Lee, T. Ahn, J.H. Kim, J.M. Ko, J.D. Kim, Nanosheets based mesoporous NiO microspherical structures via facile and template-free method for high performance supercapacitors. Electrochim. Acta 2011, 56, 4849-4857.

[9] S. Xiong, C. Yuan, X. Zhang, Y. Qian, Mesoporous NiO with various hierarchical nanostructures by quasi-nanotubes/nanowires/nanorods self-assembly: controllable preparation and application in supercapacitors. CrystEngComm. 2011, 13, 626-632.

[10] L. Wang, Y.J. Hao,Y. Zhao, Q.Y. Lai, X.Y. Xu, Hydrothermal synthesis and electrochemical performance of $\mathrm{NiO}$ microspheres with different nanoscale building blocks, Journal of Solid State Chemistry 183 (2010) 2576-2581.

[11] J.X. Zhu, Z. Gui, Y.Y. Ding, Z.Z. Wang, Y. Hu, M.Q. Zou, A Facile Route to Oriented Nickel Hydroxide Nanocolumns and Porous Nickel Oxide, J. Phys. Chem. C 2007, 111, 5622-5627.

[12] X.Y. Wang, L.J. Wan, T. Yu, Y. Zhou, J. Guan, Z.T. Yu, Z.S. Li, Z.G. Zou, Non-basic solution eco-routes to nano-scale $\mathrm{NiO}$ with different shapes: Synthesis and application, Materials Chemistry and Physics, 2011, 126, 494 499.

[13] X. Wang, L.J. Yu, P. Hu, F.L. Yuan, Synthesis of single-crystalline hollow octahedral NiO, Crystal Growth \& Design, 2007, 7, 2415-2418.

[14] A.J. Christy, M. Umadevi, Novel combustion method to prepare octahedral NiO nanoparticles and its photocatalytic activity, Materials Research Bulletin 48 (2013) 4248-4254.

[15] P. Justin, S. K. Meher, G. R. Rao, Tuning of Capacitance Behavior of NiO Using Anionic, Cationic, and Nonionic Surfactants by Hydrothermal Synthesis, J. Phys. Chem. C 2010, 114, 5203-5210.

[16] J.H. Pan, Q.Z. Huang, Z.Y. Koh, D. Neo, X.Z. Wang, Q. Wang, Scalable Synthesis of Urchinand Flowerlike Hierarchical NiO Microspheres and Their Electrochemical Property for Lithium Storage, ACS Appl. Mater. Interfaces 2013, 5, 6292-6299. 